
ASSESSING FUNCTION, QUALITY OF LIFE AND GASTROINTESTINAL INVOLVEMENT

B. Samões ${ }^{1}$, D. Fonseca ${ }^{1}$, T. Beirão ${ }^{1}$, M. Guerra ${ }^{2}$, R. Vieira ${ }^{1}$, F. Campos Costa ${ }^{1}$ T. Videira ${ }^{1}$, A. Aleixo ${ }^{1}$, P. Pinto ${ }^{1} .{ }^{1}$ Centro Hospitalar Vila Nova de Gaia/Espinho, Rheumatology, Vila Nova de Gaia, Portugal; ${ }^{2}$ Centro Hospitalar Universitário Cova da Beira, Rheumatology, Covilhã, Portugal

Background: Systemic Sclerosis (SSc) is a chronic disease with multi-organ manifestations that may contribute to disability and low quality of life. ${ }^{1}$ Therefore, anxiety and depression are more frequent in SSc patients than in general population. ${ }^{2}$ Objectives: To assess the prevalence of anxiety and depression in a SSc cohort and to evaluate its correlation with function, quality of life and assessment of gastrointestinal (GI) involvement scores.

Methods: A cross-sectional study was conducted evaluating a cohort of SSc patients. All patients answered to the Hospital Anxiety and Depression Scale (HADS) questionnaire. A cut-off score $<8$ was considered normal. Health Assessment Questionnaire (HAQ), Scleroderma HAQ (SHAQ), 36-Item Short Form Health Survey (SF-36), EuroQol-5D (EQ-5D) and University Of California, Los Angeles, Scleroderma Clinical Trials Consortium Gastrointestinal Scale (UCLA SCTC GIT) 2.0 questionnaires were also obtained. Clinical data was obtained and analyzed.

Results: We included 20 patients, 17 females [n $=17(85 \%)$ ], median (min, max) age was $52.5(28,75)$ years-old. Regarding disease classification, $13(65 \%)$ had limited SSc, $4(20 \%)$ had diffuse SSc and $3(15 \%)$ had early SSc. A score $\geq 8$ was found in $14(70 \%)$ patients on HADS-A [median (min, max) $=9(2,19)$ ] and in 12 $(60 \%)$ patients on HADS-D [median $(\min , \max )=8(1,15)]$. Depressive patients had significantly worst scores on the measures of function, such as $\mathrm{HAQ}$ and lung and gastrointestinal involvements and patient global assessment of SHAQ, of quality of life, such as EQ-5D and physical functioning, role physical, bodily pain, vitality, social functioning and mental health domains of SF-36, and on the UCLA SCTC GIT 2.0 scale. Anxious patients had significantly worst scores on social functioning and mental health domains of SF-36 and on the UCLA SCTC GIT 2.0 scale (Table 1).

Conclusion: The prevalence of depression and anxiety on SSc patients is high and should not be neglected. Overall disability and multiorgan manifestations, particularly GI involvement, may contribute to a low quality of life and consequently to depression and anxiety.

REFERENCES:

11] Firestein \& Kelley's Textbook of Rheumatology 2-Volume Set, 11th Edition

[2] Brett D. Thombs et al. Depression in Patients With Systemic Sclerosis: A Systematic Review of the Evidence. Arthritis \& Rheumatism (Arthritis Care \& Research) Vol. 57, 2007, pp 1089-1097

Table 1. Function, quality of life and gastrointestinal (GI) involvement assessment according to HADS score.

\begin{tabular}{|c|c|c|c|c|c|c|}
\hline $\begin{array}{l}\text { Results, median } \\
\text { [min, max] }\end{array}$ & $\begin{array}{c}\text { HADS-D } \geq 8 \\
(n=12)\end{array}$ & $\begin{array}{l}\text { HADS-D < } 8 \\
(\mathrm{n}=8)\end{array}$ & $\begin{array}{c}\text { P- } \\
\text { value }\end{array}$ & $\begin{array}{c}\text { HADS-A } \geq 8 \\
(n=14)\end{array}$ & $\begin{array}{c}\text { HADS-A < } \\
8(n=6)\end{array}$ & $\begin{array}{c}\text { P- } \\
\text { value }\end{array}$ \\
\hline \multicolumn{7}{|l|}{ SHAQ } \\
\hline - GI involvement & $\begin{array}{c}26.5 \\
{[0,90]}\end{array}$ & $\begin{array}{c}2 \\
{[0,40]}\end{array}$ & 0.009 & $18.5[0,90]$ & $\begin{array}{c}2.5 \\
{[0,40]}\end{array}$ & 0.091 \\
\hline - Lung involvement & $\begin{array}{c}48.5 \\
{[5,90]}\end{array}$ & $\begin{array}{c}2.5 \\
{[0,30]}\end{array}$ & 0.001 & $18[0,90]$ & $\begin{array}{c}3 \\
{[0,65]}\end{array}$ & 0.126 \\
\hline $\begin{array}{r}\text { - Patient global } \\
\text { assessment }\end{array}$ & $\begin{array}{c}67.5 \\
{[30,100]}\end{array}$ & $\begin{array}{c}4 \\
{[0,85]}\end{array}$ & 0.011 & $53.5[2,100]$ & $\begin{array}{c}41.5 \\
{[0,85]}\end{array}$ & 0.509 \\
\hline HAQ & $\begin{array}{c}1.375 \\
{[0.5,2]}\end{array}$ & $\begin{array}{c}0.1875 \\
{[0,1]}\end{array}$ & 0.001 & $1.25[0,2]$ & $\begin{array}{c}0.875 \\
{[0,1.125]}\end{array}$ & 0.147 \\
\hline EQ5D & $\begin{array}{c}0.3667 \\
{[-0.0573} \\
0.6937]\end{array}$ & $\begin{array}{c}0.6752 \\
{[0.2870,1]}\end{array}$ & 0.006 & $\begin{array}{c}0.4640 \\
{[-0.0573} \\
0.7667]\end{array}$ & $\begin{array}{c}0.6752 \\
{[0.287,1]}\end{array}$ & 0.075 \\
\hline \multicolumn{7}{|l|}{ SF36 } \\
\hline $\begin{array}{l}\text { - Physical } \\
\text { functioning }\end{array}$ & $\begin{array}{c}25 \\
{[15,75]}\end{array}$ & $\begin{array}{c}75 \\
{[50,100]}\end{array}$ & 0.001 & $40[15,100]$ & $\begin{array}{c}72.5 \\
{[25,85]}\end{array}$ & 0.106 \\
\hline - Role physical & $\begin{array}{l}31.25 \\
{[0,75]}\end{array}$ & $\begin{array}{c}72.875 \\
{[31.25,100]}\end{array}$ & 0.025 & $37.5[0,100]$ & $\begin{array}{c}65.625 \\
{[31.25,100]}\end{array}$ & 0.214 \\
\hline - Bodily pain & $41[0,74]$ & $\begin{array}{c}68 \\
{[20,88]}\end{array}$ & 0.011 & $41[0,88]$ & $\begin{array}{c}61.5 \\
{[20,74]}\end{array}$ & 0.428 \\
\hline - Vitality & $\begin{array}{c}25 \\
{[0,43.75]}\end{array}$ & $\begin{array}{c}65.625 \\
{[25,75]}\end{array}$ & 0.001 & $37.5[0,75]$ & $\begin{array}{c}65.625 \\
{[12.5,75]}\end{array}$ & 0.135 \\
\hline - Social functioning & $\begin{array}{c}37.5 \\
{[12.5,87.5]}\end{array}$ & $\begin{array}{c}87.5 \\
{[50,100]}\end{array}$ & 0.002 & $50[12.5,100]$ & $\begin{array}{c}87.5 \\
{[87.5,100]}\end{array}$ & 0.003 \\
\hline - Mental health & $\begin{array}{c}45 \\
{[25,80]}\end{array}$ & $\begin{array}{c}65.7 \\
{[51.4,85]}\end{array}$ & 0.012 & $45[25,75]$ & $\begin{array}{c}77.5 \\
{[51.4,85]}\end{array}$ & 0.005 \\
\hline UCLA SCTC GIT 2.0 & & & & & & \\
\hline - Reflux & $\begin{array}{c}0.38 \\
{[0,1.25]}\end{array}$ & $\begin{array}{c}0 \\
{[0,1.25]}\end{array}$ & 0.024 & $\begin{array}{c}0.25 \\
{[0,1.25]}\end{array}$ & $\begin{array}{c}0 \\
{[0,1]}\end{array}$ & 0.139 \\
\hline - Distension & $\begin{array}{c}1 \\
{[0.5,2]}\end{array}$ & $\begin{array}{c}0.25 \\
{[0,1.5]}\end{array}$ & 0.017 & $\begin{array}{c}1 \\
{[0.25,2]}\end{array}$ & $\begin{array}{l}0.125 \\
{[0,1]}\end{array}$ & 0.024 \\
\hline - Total & $\begin{array}{c}0.44 \\
{[0.1,0.99]}\end{array}$ & $\begin{array}{c}0.04 \\
{[0,0.97]}\end{array}$ & 0.010 & $\begin{array}{c}0.34 \\
{[0.04,0.99]}\end{array}$ & $\begin{array}{c}0.02 \\
{[0,0.44]}\end{array}$ & 0.018 \\
\hline
\end{tabular}

Disclosure of Interests: None declared

DOI: 10.1136/annrheumdis-2021-eular.1166

\section{AB0416 THE IMPACT OF FATIGUE ON SYSTEMIC SCLEROSIS PATIENTS}

B. Samões ${ }^{1}$, D. Fonseca ${ }^{1}$, T. Beirão ${ }^{1}$, M. Guerra ${ }^{2}$, R. Vieira ${ }^{1}$, F. Campos Costa ${ }^{1}$, T. Videira ${ }^{1}$, A. Aleixo ${ }^{1}$, P. Pinto ${ }^{1}{ }^{1}$ Centro Hospitalar Vila Nova de Gaia/Espinho, Rheumatology, Vila Nova de Gaia, Portugal; ${ }^{2}$ Centro Hospitalar Universitário Cova da Beira, Rheumatology, Covilhã, Portugal

Background: Fatigue is a frequent and commonly undervalued symptom among rheumatic disease, including Systemic Sclerosis (SSc). ${ }^{1,2}$

Objectives: To determine the prevalence of severe fatigue in a SSc cohort and to evaluate how it correlates with disability, quality of life and mental illness.

Methods: A cross-sectional study was conducted evaluating a cohort of SSc patients. Fatigue was evaluated using Functional Assessment Chronic IIIness Therapy (Fatigue) (FACIT-F) questionnaire. A value $<30$ was defined as severe fatigue. Health Assessment Questionnaire (HAQ), Scleroderma HAQ (SHAQ), 36-Item Short Form Health Survey (SF-36), EuroQol-5D (EQ-5D) and Hospital Anxiety and Depression Scale (HADS) questionnaires were also filled. Clinical data was obtained and analysed.

Results: We included 20 patients, 17 females [ $\mathrm{n}=17(85 \%)]$, median (min, max) age was $52.5(28,75)$ years-old. Regarding disease classification, $13(65 \%)$ had limited SSc, $4(20 \%)$ had diffuse SSc and $3(15 \%)$ had early SSc. The median FACIT-F score was $34(3,48)$. The prevalence of severe fatigue was $40 \%(n=8)$ Fatigue had a moderate negative correlation with $\mathrm{HAQ}(\tau=-0.641 ; \mathrm{p}<0.001)$ and a weak negative correlation with lung $(\tau=-0.345 ; p=0.039)$ and gastrointestinal $(\tau=-0.419 ; p=0.011)$ involvements and with patient global assessment $(\tau=$ $-0.325 ; p=0.047$ ) subtopics of SHAQ. A moderate positive correlation was found between FACIT-F and EQ-5D $(\tau=0.625 ; p<0.001)$ and physical functioning $(\tau$ $=0.560 ; p=0.001)$ and vitality $(\tau=0.777 ; p<0.001)$ domains of SF-36. The remaining SF-36 domains had a weak positive correlation with FACIT-F (Table 1). Regarding mental illness, there was a moderate negative correlation between FACIT-F and HADS-D $(\tau=-0.638 ; p<0.001)$ and HADS-A $(\tau=-0.535 ; p=0.001)$ Conclusion: Severe fatigue is frequent among SSc patients. The greater the fatigue, the greater the disability, the lower the quality of life and the worse the score on the scale of depression and anxiety.

\section{REFERENCES:}

[1] F. Basta, A. Afeltra, D.P.E. Margiotta. Fatigue in systemic sclerosis: a systematic review. Clin Exp Rheumatol 2018; 36 (Suppl. 113): S150-S160

[2] Sarah Hewlett, Emma Dures, And Celia Almeida. Measures of Fatigue. Arthritis Care \& Research Vol.63, No. S11, November 2011, pp S263-S286

Table 1. Correlation of FACIT-F with measures of disability, quality of life and mental illness

\begin{tabular}{lcc}
\hline Variables & Kendall's $\tau$ coefficient & p-value \\
\hline HAQ & -0.641 & $\mathbf{0 . 0 0 0}$ \\
SHAQ & & \\
- GI involvement & -0.345 & $\mathbf{0 . 0 3 9}$ \\
- Lung involvement & -0.419 & $\mathbf{0 . 0 1 1}$ \\
- Vascular involvement & -0.215 & 0.192 \\
- Digital ulcers & 0.102 & 0.549 \\
- Patient global assessment & -0.325 & $\mathbf{0 . 0 4 7}$ \\
EQ-5D & 0.625 & $\mathbf{0 . 0 0 0}$ \\
SF-36 & & \\
- Physical functioning & 0.560 & $\mathbf{0 . 0 0 1}$ \\
- Role physical & 0.491 & $\mathbf{0 . 0 0 3}$ \\
- Bodily pain & 0.463 & $\mathbf{0 . 0 0 6}$ \\
- General health & 0.341 & $\mathbf{0 . 0 4 5}$ \\
- Vitality & 0.777 & $\mathbf{0 . 0 0 0}$ \\
- Social functioning & 0.443 & $\mathbf{0 . 0 0 9}$ \\
- Role emotional & 0.350 & $\mathbf{0 . 0 4 1}$ \\
- Mental health & 0.376 & $\mathbf{0 . 0 2 4}$ \\
HADS-D & -0.638 & $\mathbf{0 . 0 0 0}$ \\
HADS-A & -0.535 & $\mathbf{0 . 0 0 1}$ \\
\hline
\end{tabular}

Disclosure of Interests: None declared

DOI: 10.1136/annrheumdis-2021-eular.1176

\section{AB0417 COMPARATIVE ANALYSIS OF THE FREQUENCY OF GASTRO-INTESTINAL SYMPTOMS IN THE CLINICAL EVALUATION VERSUS WITH UCLA SCTC GIT 2.0}

B. Samões ${ }^{1}$, D. Fonseca ${ }^{1}$, R. Vieira ${ }^{1}$, T. Videira ${ }^{1}$, A. Aleixo ${ }^{1}$, P. Pinto ${ }^{1}$, M. Guerra ${ }^{2}{ }^{1}$ Centro Hospitalar Vila Nova de Gaia/Espinho, Rheumatology, Vila Nova de Gaia, Portugal; ${ }^{2}$ Centro Hospitalar Universitário Cova da Beira, Rheumatology, Covilhã, Portugal 